\title{
The Reuse of (Single-Use) Cardiac Disposable of Coronary Angiography and Angioplasty: Safety and Economic Issues in Gaza Strip
}

\author{
Mohammed Habib*1, Ahmad Musran² ${ }^{2}$ and Mohammad Al Agha $^{3}$ \\ ${ }^{1}$ Department of Consultant Cardiologist, Gaza \\ ${ }^{2}$ Department of Head nurse of Cardiac Catheterization Unit, Gaza \\ ${ }^{3}$ Department of Environmental Sciences, Gaza
}

*Corresponding author: Mohammed Habib, Department of Consultant Cardiologist, Al Shifa hospital, Gaza.

Received Date: March 18, 2019

Published Date: March 27, 2019

\begin{abstract}
Background: Most countries outside the United States routinely reuse disposable medical equipment. We investigated the reuse of puncture needle, wires, sheath, manifold, inflation device, diagnostic and angioplasty catheters restored by a process strictly controlled for bioburden and sterility, in patients undergoing diagnostic coronary angiography and PCI.

Methods: We enrolled total 135 samples from different disposable (18 catheters, 6 manifold, 6 pressure line ,6 coronary wire, 6 femoral sheath, 2 inflation devices, 1 puncture needle) from 3 hospital in Gaza strip ( 45 sample from each hospital), were shipped to a central facility and were decontaminated, cleaned and tested for sterilization. The disposables were sterilized by exposure to Formaldehyde ( 90 samples,) or Cidex ( 45 samples) using a carefully validated protocol. Safety point: After sterilization disposables well rinsing with normal saline then taking swaps from outer side and inner side and were collected into a suitable container and transferred directly from collecting site to the laboratory to make culture. Cost-effective point: An average of five uses for diagnostic and three uses for angioplasty disposables.
\end{abstract}

Results: The disposable materials were collected from Aid Hospital and the European Gaza Hospital and sterilized by exposure to formaldehyde were negative results. while samples was collected from Al Shifa hospitals and sterilized by Cidex were 3 staphylococcus positive results. The procedure success rates similar to those of new products. Cost analysis suggests that An average of five uses for diagnostic disposables and three uses for angioplasty disposables may save approximately $\$ 5000$ and $\$ 11500$ respectively, per 100 procedures

Conclusion: Reuse of medical devices labelled "single use only" is common in Palestinian hospitals. Restoration of disposable of coronary angiography and angioplasty using a controlled process appears to be safe, cost saving in sterilization by Formaldehyde (but not Cidex) with success rates similar to those of new products and no detectable sacrifice in performance.

\section{Introduction}

Current guidelines generally recommend that medical devices labelled "single use only" should not be reused, but controversy arises because of their high cost and the belief that using them once only is wasteful as well as environmentally unsound [1,2]. The United States Food and Drug Administration (FDA) maintains that there is a lack of data to support the general reuse of disposable devices [3]. In 2018, 3500 coronary angioplasty procedures were performed in the Gaza Strip. At an average charge of $\$ 3,000$ per procedure. the initial hospitalization charges amounted to more than 10.5 million dollars. Although much of the disposables used for coronary angioplasty is expensive.

\section{Methods}

\section{Product cleaning}

A 135 disposable materials were collected from the cardiac catheterization laboratory after diagnostic or therapeutic coronary 
angioplasty procedures from Al Shifa Hospital, European Gaza Hospital and Public Aid Hospital each martial contains 45 samples (18 catheters: 12 diagnostic catheters and 6 guiding catheters , 6 manifold , 6 pressure line , 6 coronary wire: 2 were 0.014 inch coronary $180 \mathrm{~cm}$ guide wire and 4 were 0.035 inch $150 \mathrm{~cm}$ wire, 6 femoral sheath, 2 inflation device, 1 puncture needle) were shipped to a central facility and were decontaminated, cleaned and tested for sterilization. this Martials rinse well in potable tap water/ sterile distill water. prepared for shipment and shipped to a central facility where they were inspected, decontaminated and cleaned using normal saline. Drying to be performed by using compressed air jets free of oil. The disposables were collected from European Gaza hospital and public aid hospital were sterilized by exposure to Formaldehyde ( 90 samples,) and disposables was collected from Alshifa Hospital sterilized by exposure to Cidex (45 samples) at least 30 minus using a carefully validated protocol.

\section{Product sterilization}

Physical testing was performed using units that were cleaned, packaged and sterilized. The units were packaged in standard trays and pouches typical of those used in the industry. They are sterilized by exposure to Formaldehyde and Re-package in double layers Sterilize with ethylene oxide Label the date of re-sterilization and Note the reuse number (different color code for 1st, 2nd, 3rd, 4 th \& 5 th final reuse). Before reuse of disposables which sterilized by Formaldehyde or Cidex was cleaned again by normal saline. After sterilization the samples was cleaned, and swap were collected and transferred to Kamal Edwan Hospital Microbiology laboratory for culture.

\section{Statistics}

Earlier reports indicated that with new material, the angiographic failure rate is; $10 \%$. This pilot study was designed to have an $80 \%$ chance of detecting a $5 \%$ difference in the failure rates of new and reused catheters (i.e., alpha 0.05), the difference between culture in formaldehyde and Cidex group was compare by $\mathrm{X}$ square test.

\section{Results}

A 135 disposable materials were collected from the cardiac catheterization laboratory after diagnostic or therapeutic coronary angioplasty procedures from Al Shifa Hospital, European Gaza Hospital and Public Aid Hospital, each martial contains 45 samples. The disposables were collected from European Gaza hospital and public aid hospital were sterilized by exposure to Formaldehyde (90 samples,) and disposables was collected from Alshifa Hospital sterilized by exposure to Cidex ( 45 samples) at least 30 minus using a carefully validated protocol.

Safety point: The culture from the disposables were collected from European Gaza hospital and public aid hospital were sterilized by exposure to Formaldehyde was negative. The culture from disposables was collected from Alshifa Hospital sterilized by exposure to Cidex at least 30 minus was 3 staph positive (P: 0.016). There were no episodes of catheter fracture or of pyrogen reactions occurring in patients in the trial. Table $1(a, b)$ samples numbers of sterilized by exposure to Formaldehyde or Cidex and swap culture:
Table 1A:

\begin{tabular}{|c|c|c|}
\hline Tool Name & $\begin{array}{c}\text { Cidex } \\
\text { N:45 }\end{array}$ & $\begin{array}{c}\text { Formal } \\
\text { aldehyde N: 90 }\end{array}$ \\
\hline Guiding catheter Fr 6 & 6 & 12 \\
\hline Coronary wire 0.014-inch, 182cm & 2 & 4 \\
\hline Puncture needle & 1 & 2 \\
\hline Coronary Guide wire J TIP 0.035 - 150cm & 4 & 8 \\
\hline Femoral sheath F6 11cm (Kit) & 6 & 12 \\
\hline Inflation device & 2 & 24 \\
\hline Diagnostic catchers & 12 & 12 \\
\hline Manifold 3 way & 6 & 12 \\
\hline Pressure line $100 \mathrm{~cm}$ & 6 & 4 \\
\hline
\end{tabular}

Table 1B:

\begin{tabular}{|c|c|}
\hline & Sigle Use \\
\hline Diagnostic coronary & \\
\hline angiography devices/procedure & \\
\hline Right and left diagnostic catheters & $35 \$$ \\
\hline Femoral sheath & $15 \$$ \\
\hline 0.035 & $15 \$$ \\
\hline inch wire, 180cm & $13 \$$ \\
\hline 3 way manifold/pressure line & $2 \$$ \\
\hline Punctured needle & $85 \$$ \\
\hline Total & \\
\hline Percutaneous Coronary Intervention Devices/ \\
Procedure & $60 \$$ \\
\hline Guiding catheter & $15 \$$ \\
\hline 0.035 inch wire, 180cm & $65 \$$ \\
\hline 0.014 inch coronary wire & $50 \$$ \\
\hline Inflation device & $13 \$$ \\
\hline 3 way manifold/pressure line & $205 \$$ \\
\hline Punctured needle & \\
\hline Total & \\
\hline
\end{tabular}

\section{Cost effective point}

We calculated an average of five uses for diagnostic and three uses for angioplasty disposables.

\section{Diagnostic coronary angiography procedure}

a. Total Cost of new disposable (\$): 85 \$

b. Total Cost of rasterized for five uses for devices/ procedure: $17 \$$

c. Sterilization procedure cost and packaging/ for procedure: $8 \$$

d. Cost for 100 procedures with new disposables $=8500 \$$

e. Calculated An average of five uses for diagnostic:

f. Cost for 100 procedures with rasterized device $=8500 \$+$ $(25 \times 5 \times 100) / 6=3500$

g. Total cost cost savings per 100 procedure: $8500-3500=$ 5000 \$ 


\section{Percutaneous coronary intervention procedure}

a) Total Cost of new disposables (\$): $205 \$$

b) Total Cost of rasterized for five uses for devices/ procedure: $41 \$$

c) Sterilization procedure cost and packaging/procedures: 9 $\$$

d) Cost for 100 procedures with new device $=20500 \$$

e) Calculated An average of three uses for PCI disposables

f) Cost for 100 procedures with rasterized device $=20500 \$$ $+(50 \times 3 \times 100) / 4=8875$

g) cost savings per 100 procedure: $20500-8875=11625 \$$

\section{Discussion}

Our study suggested no any bacterial growth in culture from the disposables were collected from European Gaza hospital and public aid hospital were sterilized by exposure to Formaldehyde. And 3 positive samples of staphylococcus was detected in culture from disposables were collected from Al shifa Hospital sterilized by exposure to Cidex. So that we don't recommend using Cidex to sterilize diagnostic and PCI disposables. Other prospective studies were performed by Frank et al. [4] in the period of November 1986 to June 1987 involving 414 consecutive adult patients submitted to heart catheterize utilizing both new and recycled catheters. After the procedure the axillary temperature was measured two times per day and the catheter entry site was examined daily. Fever was defined as an axillary temperature higher than $37.4^{\circ} \mathrm{C}$. The heart catheters were processed as follows: immediately after the procedure, the catheters were rinsed in tap water for 10 minutes; subsequently they were disinfected using 3\% Gigasept (an aldehyde) and were placed in a solution containing detergent for 1 hour. Following this they were again rinsed in tap water for 10 minutes and dried using compressed air. Finally, they were sterilized in ethylene oxide (15\% ethylene oxide and 85\%carbon dioxide) for 45 minutes. Before reusing, the catheters were aired at room temperature for 14 days. The incidence of fever in the patients who used new catheters was 4.4\% (7/158); 4.7\% (7/151) for patients who used recycled catheters reused once or twice and $6.0 \%(6 / 100)$ for recycled catheters reutilized more times. This difference was not statistically significant. Some descriptive studies related to the occurrence of infectious diseases after heart catheterize have been published. Although they are studies with limited power of evidence, these studies point out some problems related to this type of procedure. Sande et al. [5] evaluated 106 patients to detect bacteremia after this procedure. They found $8.0 \%(3 / 38)$ positive blood tests that the authors interpreted as contaminates of the skin. In this study no cases of bacteremia were identified. Shawker et al. [6] studied 100 heart catheterizations and detected 23 patients with positive blood cultures. Of these 11 cases were considered to be contaminants of the skin or air and in 12 patients gram negative bacteria were detected. Transitory bacteremia was detected in four cases. The authors attributed this finding to a failure in the cleaning process. Although experimental and clinical data suggest that catheter reuse may not be associated with an increased risk of morbidity and mortality to patients, including the risk of equipment breakage [7], particulate body embolization [8], infection [9], endotoxic reaction and toxicity, the cost-benefits of catheter reuse strategies have not yet been closely evaluated. It has been assumed that result would be associated with substantial cost savings. Our study was designed to determine the potential for cost savings An average of five uses for diagnostic disposables and three uses for angioplasty disposables may save approximately $\$ 5000$ and $\$ 11500$ respectively, per 100 procedures.

\section{Conclusion}

Restoration of disposable of coronary angiography and angioplasty balloon catheters to the manufacturer's original specifications results in a high quality product that can be used alone or with adjunctive devices on all types of coronary angioplasty lesions. Operators should expect to achieve success rates equivalent to those published with new coronary angiography and angioplasty disposables. There are three concerns related to safety with the reuse of disposable the efficiency of cleaning and sterilization, the effects of cleaning, disinfection and sterilization on the chemical, physical and mechanical integrity.

\section{Acknowledgement}

None.

\section{Conflict of Interest}

No conflict of interest.

\section{References}

1. Lacroix D, Lucas H, Stewart I (1994) Anxiety, misinformation and greed. Aust Nurs J 2: 17-20.

2. Campbell BA, Wells GA, Palmer WN, Martin DL (1987) Reuse of disposable medical devices in Canadian hospitals. Am J Infect Control 15: 196-200.

3. (1987) United States Food and Drug Administration. Compliance Policy Guide. Reuse of medical disposable devices. Washington, Pp. 7124.6.

4. Frank U, Herz L, Daschner FD (1988) Infection risk of cardiaccatheterization and arterial angiography with single and multipleuse disposable catheters. Clin Cardiol 11(11): 785-787.

5. Sande MA, Levinson ME, Lukas DS, Kaye D (1969) Bacteremiaassociated with cardiac catheterization. N Engl J Med 281(20): 1104-1106.

6. Shawker TH, Kluge RM, Ayella RJ (1974) Bacteremia associated with angiography. JAMA 229(8): 1090-1092.

7. Bentolila P, Jacob R, Roberge F (1990) Effects of m-use on the physkal characteristia of aogmgmphii catheters. J Med Eng Techaoll 14: 254-259.

8. Bourassa MG, Cantin M, saaban EB, Pedmsmt E (1975) Scatming electrontnicmqy of wface irregularities aad thrombogewis of polyurethane and pdyethyieene coronary catheters. Circulation 53: 2-6.

9. Rain CE, Koelder PR (1977) Reuse of disposable catheters and guide wires. Radiology 122: 577-579. 